\title{
Electrochemotherapy in treatment of tumours
}

\author{
G. Sersa ${ }^{\text {a }}$, D. Miklavcic ${ }^{\text {b }}$, M. Cemazar ${ }^{a}$, Z. Rudolf ${ }^{a}$, G. Pucihar ${ }^{\text {b }}$, M. Snoj ${ }^{\text {a,* }}$ \\ ${ }^{a}$ Institute of Oncology Ljubljana, Zaloska 2, SI-1000 Ljubljana, Slovenia \\ ${ }^{\mathrm{b}}$ University of Ljubljana, Faculty of Electrical Engineering, Trzaska 25, SI-1000 Ljubljana, Slovenia
}

Accepted 29 May 2007

Available online 5 July 2007

\begin{abstract}
Aim: Electrochemotherapy is a local drug delivery approach aimed at treatment with palliative intent of cutaneous and subcutaneous tumour nodules of different histologies. Electrochemotherapy, via cell membrane permeabilising electric pulses, potentiates the cytotoxicity of nonpermeant or poorly permeant anticancer drugs with high intrinsic cytotoxicity, such as bleomycin or cisplatin, at the site of electric pulse application.

Methods: An overview of preclinical and clinical studies is presented, and the treatment procedure is further critically evaluated.

Results: In clinical studies electrochemotherapy has proved to be a highly efficient and safe approach for treating cutaneous and subcutaneous tumour nodules. The treatment response for various tumours (predominantly melanoma) was $\sim 75 \%$ complete and $10 \%$ partial response of the treated nodules.

Conclusions: Electrochemotherapy is a new, clinically acknowledged method for the treatment of cutaneous and subcutaneous tumours. Its advantages are high effectiveness on tumours with different histologies, simple application, minimal side effects and the possibility of effective repetitive treatment.
\end{abstract}

(C) 2007 Elsevier Ltd. All rights reserved.

Keywords: Electroporation; Electrochemotherapy; Bleomycin; Cisplatin; Cutaneous tumours

\section{Introduction}

Several novel tumour-targeting and drug delivery approaches in cancer treatment are currently undergoing intensive investigation in order to increase the therapeutic index, among them physical approaches such as tissue electroporation. Electroporation of tissue increases the membrane permeability of cells, specifically in the area that is exposed to the applied electric pulses. ${ }^{1,2}$

In the past 25 years, electroporation has proved to be effective in facilitating the transport of different molecules across the plasma membrane. By using short intense electric pulses, the plasma membrane becomes permeable to molecules otherwise deprived of membrane transport mechanisms. When a cell is exposed to an electric field, for instance when electric pulses are applied to the tissue, due to its geometrical and material properties, transmembrane potential is induced across the cell plasma membrane (Fig. 1). If the induced transmembrane potential is

\footnotetext{
* Corresponding author. Tel.: +386 15879 518; fax: +386 15879434 . E-mail address: msnoj@onko-i.si (M. Snoj).
}

sufficiently high, structural changes leading to increased membrane permeability are induced. Although the exact mechanism operating at the molecular level and the various structures has not yet been fully elucidated, a flow of molecules was demonstrated through areas of the membranes in regions where the highest absolute value of the induced transmembrane potential was observed after exposure of cells to electric pulses (Fig. 2). The use of square-wave electric pulses of suitable duration and amplitude preserves the viability of cells, allowing for resealing of the cell membrane a few minutes after the application of electric pulses. $^{1-4}$

Electroporation-based cancer treatment approaches are currently undergoing intensive investigation in the field of drug delivery and gene therapy. Their first biomedical application in the treatment of cancer came in the form of electrochemotherapy, which, since its beginnings in the late 1980s, has evolved into a clinically verified treatment approach for cutaneous and subcutaneous tumour nodules. ${ }^{5-7}$ Electrochemotherapy is defined as a local treatment which, via cell membrane permeabilising electric pulses, potentiates the cytotoxicity of non-permeant or poorly 

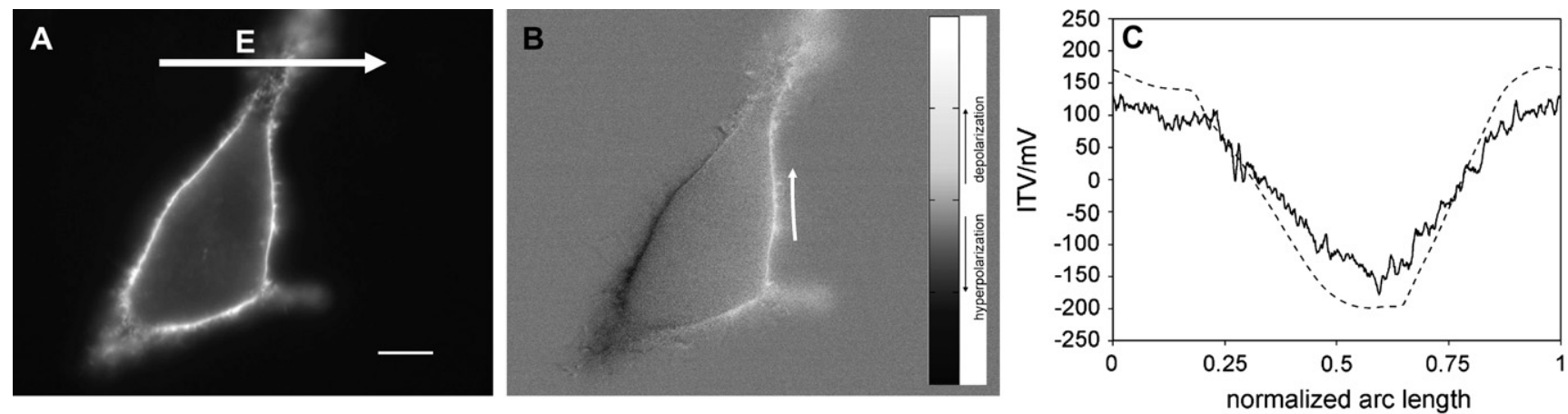

Figure 1. Transmembrane potential induced on a melanoma cell and measured with potentiometric dye di-8-ANEPPS. (A) Fluorescence image of a cell acquired during electric field exposure $(100 \mathrm{~V} / \mathrm{cm}$ ). Bar represents $10 \mu \mathrm{m}$. (B) Changes in fluorescence of the cell obtained by subtracting the control image (not shown) from the image with pulse. White arrow shows the path along which the potential was measured. (C) The potential along the perimeter of the cell. Solid curve, measured values; dashed curve, numerically calculated values (numerical model not shown). ${ }^{3}$

permeant anticancer drugs with high intrinsic cytotoxicity at the site of electric pulse application.

\section{In vitro and in vivo studies}

Since electroporation can facilitate drug transport through the cell membrane only for poor or non-permeant molecules, suitable candidates for electrochemotherapy are limited to those drugs that are hydrophilic and lack transport systems in the membrane. Several chemotherapeutic drugs were tested on cells for potential application in combination with electroporation; these include daunorubicin, doxorubicin, etoposide, paclitaxel, actinomycin $\mathrm{D}$, adriamycin, mitomycin C, 5-fluorouracil, vinblastine, vincristine, gemcitabine, cyclophosphamide, carboplatin, cisplatin and bleomycin. Electroporation of cells increases the cytotoxicity of some of these drugs anywhere from 1.1 up to several thousandfold. However, only two of these drugs have been identified to date as potential candidates for electrochemotherapy of cancer patients: bleomycin and cisplatin. $^{4}$
The transport of bleomycin across the non-permeabilised plasma membrane is achieved by carrier proteins that internalise it via the endocytotic pathway. This process is limited by the low number of carrier proteins exposed at the cell surface, as well as by withdrawal of these proteins from the membrane due to endocytosis. Hence, this mechanism limits the uptake of bleomycin and its cytotoxicity. ${ }^{4,8}$ The exposure of cells to electric pulses, and consequent membrane electroporation leading to increased membrane permeability, enables the direct access of bleomycin to cytosol and transport to DNA, where a few hundred molecules are needed for its cytotoxicity. Therefore, electroporation of cells potentiates the cytotoxicity of bleomycin up to several thousandfold, as reported in the initial study by Mir and confirmed by several consecutive studies. ${ }^{4,9}$

Cisplatin transport through the non-permeabilised plasma membrane is also hampered. Only $50 \%$ of cisplatin is transported through the plasma membrane by passive diffusion, whereas the rest is transported by carrier molecules. The overall flux across the plasma membrane is thus limited. Electroporation of the plasma membrane enables increased flux and accumulation of the drug in cells, which
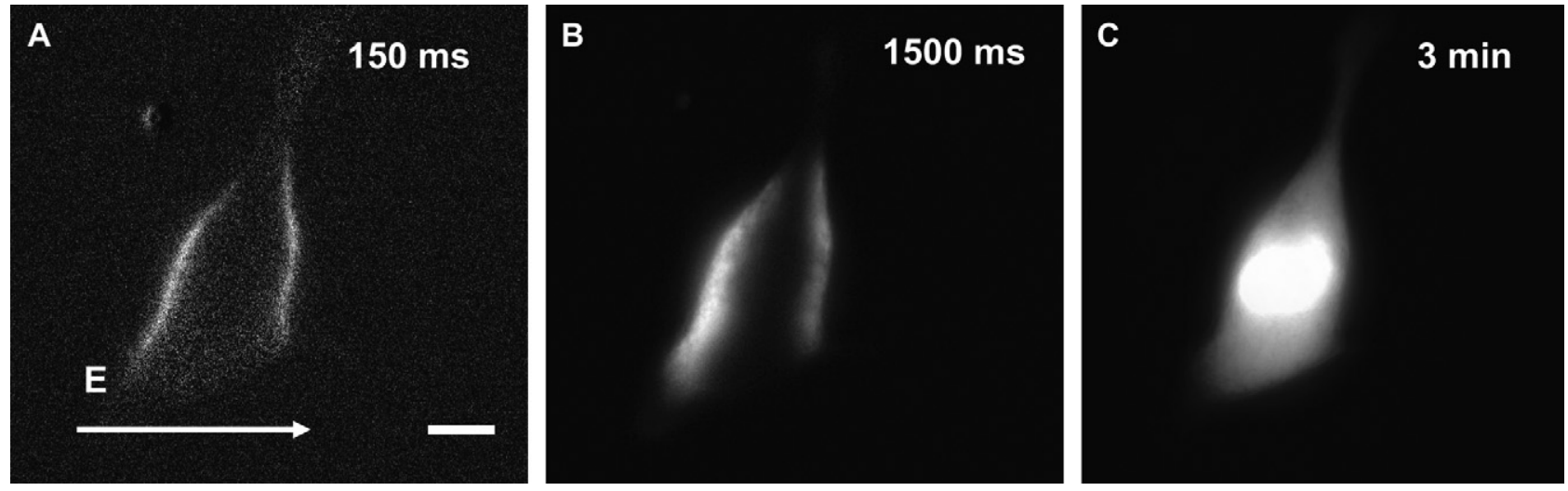

Figure 2. Visualisation of regions of the membrane where electroporation-mediated transport of molecules occurs. (A) Fluorescence of the cell at $150 \mathrm{~ms}$, (B) $1500 \mathrm{~ms}$, and (C) $3 \mathrm{~min}$ after pulse delivery. The cell was exposed to a single $(650 \mathrm{~V} / \mathrm{cm})$ rectangular electric pulse. A fluorescent marker, propidium iodide, was added to the cells before the electric pulse was applied to visualise the permeabilised regions of the cell membrane. Bar represents $10 \mu \mathrm{m}$. 
results in an increase of cisplatin cytotoxicity by up to 80 -fold. ${ }^{10}$ This increase in cisplatin uptake and DNA adduct generation is significant; however, it is still lower than in the case of bleomycin. ${ }^{4}$

Due to the observed increase in bleomycin and cisplatin cytotoxicity and the fact that bleomycin and cisplatin are commonly used drugs, further in vivo studies evaluating the antitumour effectiveness of electrochemotherapy have concentrated predominantly on these two drugs.

Extensive in vivo studies were performed on different animal tumour types, either transplantable or spontaneous. High antitumour effectiveness of electrochemotherapy was demonstrated on fibrosarcomas, melanoma, and carcinomas in mice, rats and rabbits; good clinical results were also obtained in veterinary medicine on cats, dogs and horses. ${ }^{11-15}$ In these studies solid subcutaneous tumours in the muscle, liver or brain, being either sarcomas, carcinomas, melanoma or neuroblastoma, were used to demonstrate the antitumour effectiveness of electrochemotherapy. Also, the drug administration route (intravenous or intratumoural), drug dosage, timing of its administration and electrical parameters were elaborated. It was determined that the drugs may be injected either intravenously or intratumourally and electric pulses applied to the tumours within a few minutes thereafter ( 8 electric pulses; amplitude over distance $1300 \mathrm{~V} / \mathrm{cm}$, duration $100 \mu \mathrm{s}$; frequency $1 \mathrm{~Hz}$ ). In these studies it was demonstrated that with drug doses that have minimal or no antitumour effectiveness, high (up to 75\%) complete responses of the electrochemotherapy-treated tumours were obtained. ${ }^{4,10,11}$ The drug doses used were so low that they had no systemic toxicity. Also the application of electric pulses to the tumours had no antitumour effectiveness and no systemic side effects. Local side effects were contractions of the muscles underlying the treated area, but muscle contractions and associated pain are present only during the application of electric pulses.

\section{Active mechanisms of electrochemotherapy}

The principal mechanism of electrochemotherapy is the electroporation of cells in tumours, which increases drug effectiveness by enabling the drug to reach the intracellular targets. This increased drug uptake in tumours has been demonstrated for bleomycin and cisplatin: the accumulation of these two drugs in tumours was increased two to fourfold, compared to tumours without electroporation. ${ }^{16,17}$ Furthermore, a twofold increase in cisplatin DNA adducts was determined in electroporated tumours. ${ }^{17}$

Besides membrane electroporation, the application of electric pulses to tissues induces a transient but reversible reduction of blood flow. ${ }^{18}$ The $80 \%$ decrease in tumour blood flow immediately following the application of electric pulses induces drug entrapment in the tissue for several hours, providing more time for the drug to act. ${ }^{19}$ Tumour blood flow returns to normal in tumours within $24 \mathrm{~h}$ after the application of electric pulses; whereas in normal tissue the restoration is faster, within several hours. ${ }^{4,18,20}$

The cytotoxic effect of electrochemotherapy is not limited only to tumour cells in the tumours. Electrochemotherapy acts also on stromal cells, including endothelial cells in the lining of tumour blood vessels, resulting in their death, abrogation of tumour blood flow and consequently a cascade of tumour cell death surrounding the vessels. This vascular disrupting mechanism of electrochemotherapy contributes to its antitumour effectiveness and has been demonstrated for both bleomycin and cisplatin. ${ }^{19,21}$

Differences in the antitumour effectiveness of electrochemotherapy were observed between immunocompetent and immunodeficient experimental animals, indicating the involvement of immune response in antitumour effectiveness. The observed tumour growth delay in immunocompetent mice was twice as long as in immunodeficient mice, and a high percentage ( $80 \%$ ) of tumours were cured, whereas none were cured in immunodeficient mice. ${ }^{22}$ Due to massive tumour antigen shedding in organisms after electrochemotherapy, systemic immunity is induced and can be upregulated by additional treatment with biological response modifiers such as IL-2, IL-12, GM-CSF and TNF- $\alpha .^{4,23,24}$

\section{Clinical studies}

\section{History}

The first clinical study was published in 1991 on head and neck tumour nodules, ${ }^{5}$ which was thereafter followed by several others. ${ }^{25-29}$ Most frequently single or multiple cutaneous or subcutaneous melanoma nodules were treated but nevertheless response was demonstrated in breast and head and neck cancer nodules, as well as Kaposi's sarcoma, hypernephroma, chondrosarcoma and basal cell carcinoma. ${ }^{6}$ The studies were conducted on either recurrent or primary tumour nodules in one treatment session or repetitive sessions till tumour response. An extensive and detailed review on the results of these phase II studies, according to the tumour type, drug used in electrochemotherapy and cancer centre has recently been published elsewhere and is beyond the scope of the present publication. ${ }^{6}$ For the purpose of the present article the results of those trials have been condensed in Table 1 as studies before ESOPE trial.

In brief, in melanoma, electrochemotherapy with bleomycin given intravenously resulted in $45 \%$ complete responses

Table 1

Clinical response to electrochemotherapy in the ESOPE clinical trial and previous clinical studies

\begin{tabular}{lcclllll}
\hline Electrochemotherapy & Patients & Nodules & \multicolumn{6}{l}{ Response $(\%)$} \\
\cline { 3 - 7 } & & & PD & NC & PR & CR & OR \\
\hline Before ESOPE study & 247 & 1009 & 6 & 11 & 19 & 64 & 83 \\
ESOPE study & 41 & 171 & 5 & 10 & 11 & 74 & 85 \\
\hline
\end{tabular}


whereas given intratumourally in $77 \%$ complete responses. After electrochemotherapy with cisplatin the compete response rate was $67 \%$ when cisplatin was administered intratumourally and $48 \%$ when administered intravenously. In non-melanoma tumours (squamous cell carcinomas of head and neck, basal cell carcinoma of the skin, breast adenocarcinoma, salivary gland adenocarcinoma, hypernephroma) similar results were obtained: electrochemotherapy resulted in $53 \%$ complete response rate when bleomycin was given intravenously; $63 \%$ complete responses when bleomycin was given intratumourally; and 58\% complete responses when cisplatin was given intratumourally.

\section{ESOPE trial}

However, these clinical studies were performed with slightly variable treatment protocols, different electrodes and different electric pulse generators. Thus there was a need for a prospective non-randomised multi-institutional study. This study was conducted by a consortium of four cancer centres gathered in the ESOPE project funded under the European Commission's 5th Framework Programme. Treatment response after electrochemotherapy according to tumour type, drug used, route of its administration and type of electrodes was tested. ${ }^{30}$ The results of this study can be summarised as follows:

- An objective response ${ }^{31}$ rate of $85 \%$ (73.7\% complete response rate) was achieved for electrochemotherapytreated tumour nodules, regardless of tumour histology and drug or route of administration used. The cutaneous and subcutaneous nodules of melanoma were treated predominantly, breast cancer, colon cancer, squamous cell carcinoma of the skin, squamous cell carcinoma of cervix, Kaposi and leiomyosarcoma cutaneous and subcutaneous tumour nodules were treated too in decreasing sequence.

- At 150 days after treatment, the local tumour control rate for electrochemotherapy was $88 \%$ with bleomycin given intravenously, $73 \%$ with bleomycin given intratumourally and $75 \%$ with cisplatin given intratumourally, demonstrating that all three approaches were equally effective in local tumour control.

- Treatment of tumour nodules located in previously irradiated areas was equally as effective as those that were not in previously irradiated areas (objective response rate $88.2 \%$ vs. $81.4 \%$, respectively).

- Side effects of electrochemotherapy were minor and tolerable (muscle contractions and pain sensation). The acceptability of the treatment was demonstrated by interviewing the patients; $93 \%$ of them responded that they would be willing to accept the treatment next time if it would be indicated.

In all clinical studies reported to date, including the ESOPE study, 288 patients have been treated: 782 tumour nodules were treated by electrochemotherapy with bleomycin and 398 tumour nodules were treated by electrochemotherapy with cisplatin. The results of the ESOPE study are comparable to previously reported results on the effectiveness of electrochemotherapy (Table 1)..$^{6,30}$

\section{Limitations of electrochemotherapy}

Electrochemotherapy is a local treatment, which has been mainly used when other modalities of local tumour control have failed. Its main drawback is that it cannot be used for the treatment of deep tumours, because currently only electrodes for treatment of cutaneous and subcutaneous tumours have been designed and produced. Furthermore, there is a lack of comparative studies with other local treatments, such as radiotherapy and isolated limb perfusion, which should obviously be initiated. For safety reasons electrochemotherapy is not recommended in patients with cardiac pacemakers and patients on anticoagulant therapy.

\section{Treatment procedure}

Electrochemotherapy is a procedure that combines a membrane non-permeant or poorly permeant cytotoxic drug having an intracellular target, such as bleomycin or cisplatin, with the application of short intense electric pulses causing increased membrane permeability. The prerequisite for effective electrochemotherapy is a sufficient drug concentration and distribution within the tumour, as well as an adequate electric field distribution (Fig. 3). Therefore exact procedures for either systemic or local drug delivery, using either bleomycin or cisplatin, followed by the application of electric pulses, need to be followed for each specific clinical condition. Hence, standard operating procedures (SOP) using an electric pulse generator, Cliniporator (IGEA, s.r.l. Carpi, Modena, Italy), have recently been published. ${ }^{33}$

\section{Injection site, timing and drug dosage}

The key issue in electrochemotherapy is to assure that the drug is present in the tumour when electric pulses are applied. Bleomycin can be delivered intratumourally or systemically, whereas cisplatin should be injected intratumourally to achieve the best results. When the drug is delivered systemically, electric pulses need to be delivered to the tumour site during the pharmacokinetic peak, which was reported to be between 8 and 28 min in humans; ${ }^{34}$ for intratumoural application, however, the pulses need to be delivered from $1 \mathrm{~min}$ to $10 \mathrm{~min}$ after drug injection. ${ }^{17,26}$ The dose for bleomycin intravenous injection is $15000 \mathrm{IU} / \mathrm{m}^{2}$, while for intratumoural injection the dose for bleomycin is approx. $500 \mathrm{IU} / \mathrm{cm}^{3}$ and for cisplatin approx. $1 \mathrm{mg} / \mathrm{cm}^{3}$, depending on the tumour volume. ${ }^{33}$ 


\section{Electrochemotherapy}

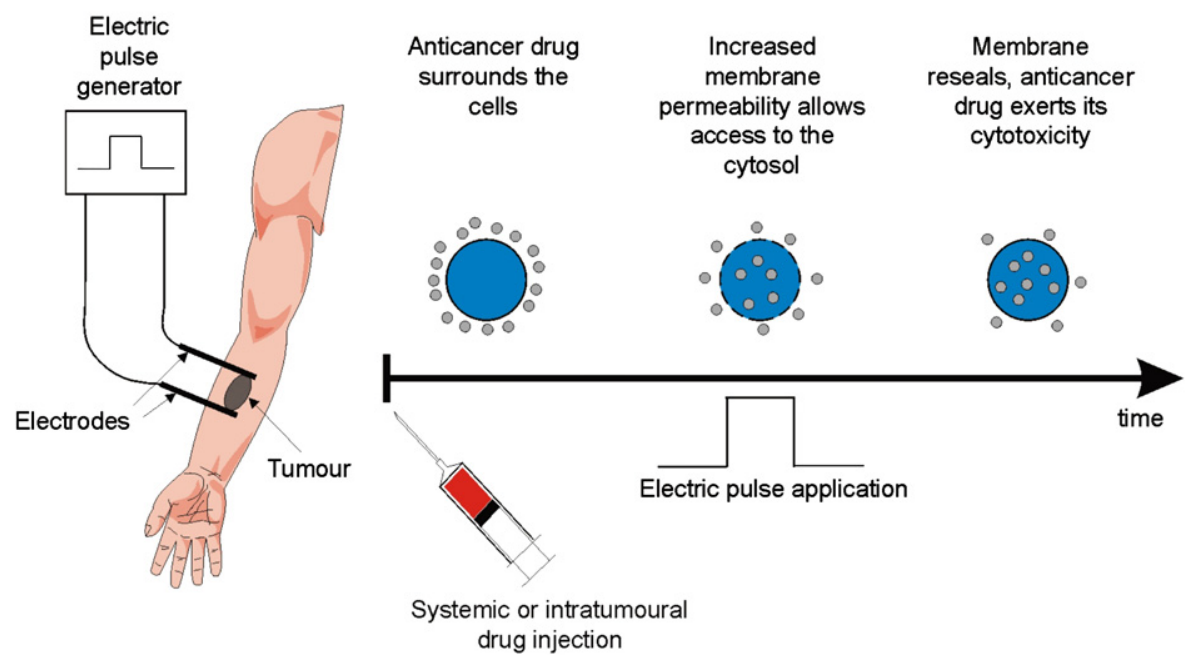

Figure 3. Principle of electrochemotherapy. Non-permeant or poorly permeant anticancer drug with intracellular target, injected either systemically or intratumourally, surrounds tumour cells. Application of adequate electric pulses causes an increase in membrane permeability, which allows for the entrance of anticancer drug into the cells to exert its cytotoxic action (redrawn from $^{32}$ ).

\section{Characteristics of electric pulses}

The electric pulses delivered were in most reported cases eight pulses of $100 \mu$ s duration delivered at $1 \mathrm{~Hz}$, or more recently $5 \mathrm{kHz}$ repetition frequency. ${ }^{6,30}$ These pulses proved to be optimal for the cell penetration of "small drugs", such as bleomycin and cisplatin, provided that the local electric field established in the target tissue, i.e. tumour, was sufficiently high. Such an electric field can be obtained at amplitudes above $1000 \mathrm{~V}$ for parallel plate electrodes with a distance of $8 \mathrm{~mm}$. The local field in the target tissue, however, depends on electrode geometry and electrode placement. Thus the choice and placement of electrodes need to be carefully selected with respect to the shape and size of the tumour, and appropriate pulse amplitudes need to be selected as well. ${ }^{1}$

\section{Types of electrodes}

Although there are different types of electrodes available on the market, ${ }^{35}$ in principle two different types of electrodes exist: plate electrodes and needle electrodes. Plate electrodes are used for the treatment of skin or superficial lesions (Fig. 4). The depth of penetration of the effective electric field is rather small, and it depends on the distance between the electrodes: the greater the distance, the deeper the penetration of the electric field into the tissue, given that a larger voltage needs to be applied between both electrodes.

Needle electrodes are of two kinds: needles are positioned either in two parallel rows or in a circular (hexagonal) array. In contrast to plate electrodes, needle electrodes must be inserted throughout the tumour tissue up to the deep tumour border (Fig. 4). ${ }^{1}$
Regardless of the type of electrode, whether plate or needle, the electric field is highest around the electrode and between the electrodes, but drops off very rapidly outside the electrode array (Fig. 4). Thus, if the tumour is larger than the distance between the electrodes, the entire tumour can be efficiently treated by moving and placing electrodes adjacently for each consecutive electric pulse application. Because of the structural heterogeneity of tissues, including tumours, the electric field which should be established in tumours for the permeabilisation of cells is difficult to determine. ${ }^{36}$ It is therefore critical to apply the suggested pulse amplitude for different types of electrodes, as provided by the manufacturers of clinical electroporators.

Single or multiple tumour nodules can be treated, even up to 100 nodules can be treated in one electrochemotherapy session. ${ }^{37}$ Single treatment can result in complete eradication of tumour nodules with up to several years remission, however if the treatment is not effective the treatment could be repeated at monthly intervals. Bigger tumours of more than $3 \mathrm{~cm}$ in size can be successfully treated by repetitive application of electric pulses to the tumour until the whole tumour area is covered. By using needle electrodes tumours of up to $2 \mathrm{~cm}$ in thickness can be treated.

\section{Clinical uses}

Electrochemotherapy has proved to be effective in various cutaneous tumours. ${ }^{6,30}$ Currently, its main use is as a palliative treatment of cutaneous and subcutaneous tumour nodules with different histologies after all other treatment modalities have failed or proved insufficient. Most frequently it is used in the treatment of multiple 

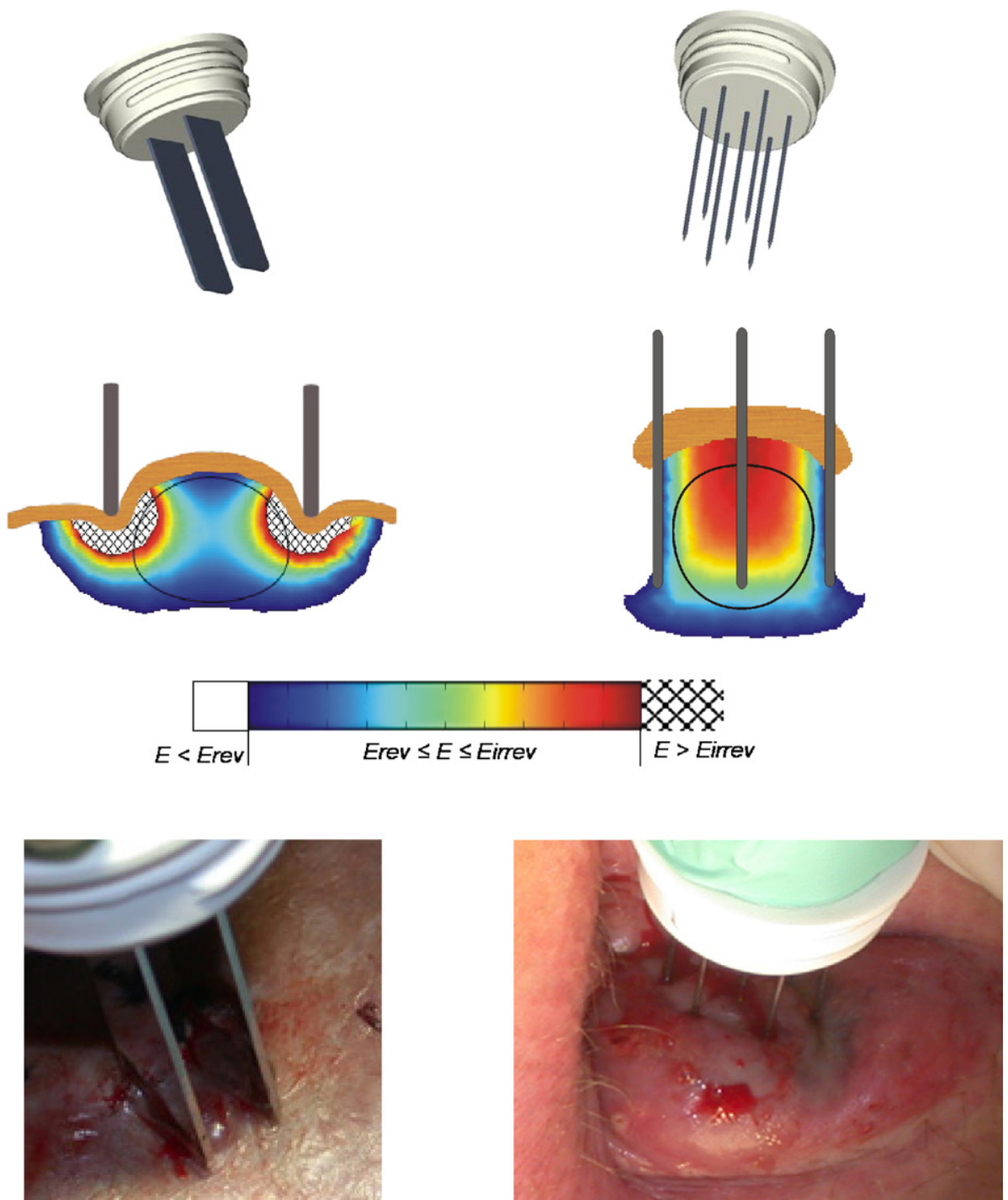

Figure 4. Types of electrodes, electric field distribution and application of electric pulses to tumours. Plate (left) or needle electrodes (right) are used noninvasively and invasively, respectively. The application of high voltage electric pulses via plate electrodes has a different electric field distribution compared to needle electrodes. Plate electrodes are more appropriate for tumours on the surface, not situated deep in the tissue. Needle electrodes have an electric field distribution that is predominantly limited to the area between the electrodes and to the tips of the electrodes, so that the needle needs to be inserted at least to the entire depth of the tumour. In both cases larger tumours can be treated by repositioning the electrodes so that the entire tumour volume is totally covered by a sufficiently high electric field.

cutaneous metastases of melanoma when they cannot be surgically excised due to their number or localisation. In such cases long-term remission-up to several years-can be obtained (Fig. 5). ${ }^{27,29,38}$

Electrochemotherapy can also be used as a cytoreductive treatment before surgical resection in an organ-sparing treatment attempted. It has been used in such a setting before a sphincter-sparing resection of anal melanoma ${ }^{39}$ and in digital chondrosarcoma, rescuing the finger from amputation. ${ }^{40}$

Furthermore, it could be used to treat basal cell carcinoma on the face with the intent to obtain a cure. Its beneficial antitumour effects have been proved, giving better cosmetic results than excisional surgery. ${ }^{41}$

\section{Current development and future medical applications of tissue electroporation}

Since electrochemotherapy with bleomycin or cisplatin has been shown to act synergistically with radiotherapy in preclinical studies, its use can be foreseen for the radiosensitisation of cutaneous tumours, predominantly in the palliative treatment of progressive disease. ${ }^{42,43}$

Further development is focused on the treatment of internal tumours by development of endoluminal electrodes. This technological development could be used either for electrochemotherapy or gene electrotransfer. ${ }^{44}$

The use of electroporation technology in gene therapy is termed gene electrotransfer. Although this approach needs 

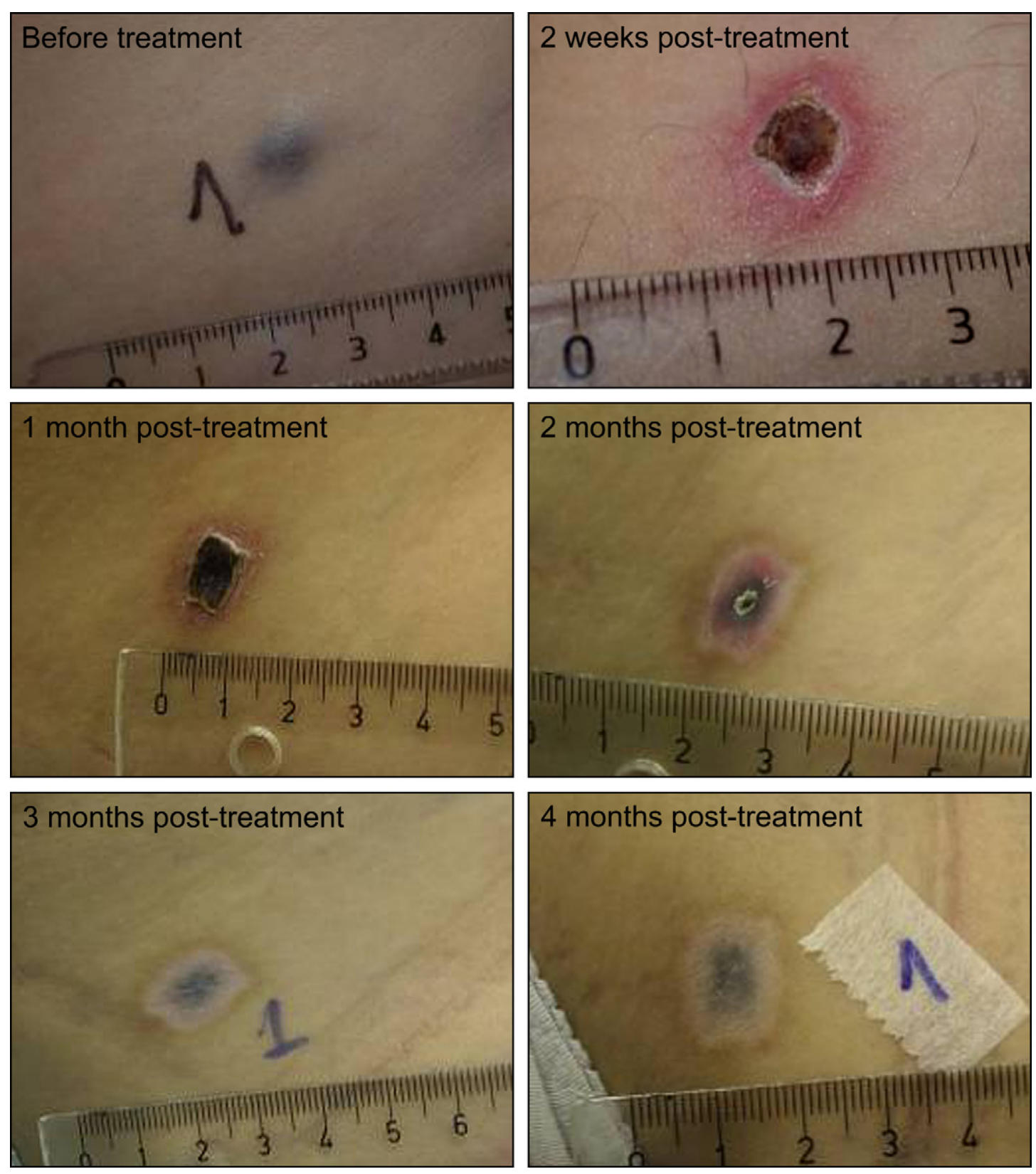

Figure 5. Typical response of melanoma nodule after treatment with electrochemotherapy. A scab forms after treatment, which exfoliates after approximately 2 months. In the treated area the pigmentation remains for a long time.

further development in order to achieve better electrotransfer of different kinds of nucleic acids (siRNA, plasmid DNA, oligonucleotides), its clinical use can be foreseen in the near future. ${ }^{45,46}$ The first phase I/II clinical studies are ongoing. ${ }^{47}$

In electrochemotherapy, electroporation is used as a means for the delivery of cytotoxic drugs into cells, with the intent of eradicating cancer cells. However, the application of electric pulses with higher amplitudes ${ }^{48}$ or nanopulses, ${ }^{49}$ which leads to the irreversible electroporation of cells and consequently to cell death, was suggested to be used instead of electrochemotherapy for tumour ablation. This technology is still under development, though.
The tumour blood modifying effect of electroporation could be used for activation of bioreductive drugs specifically in tumours, as has already been demonstrated for tirapazamine. ${ }^{50}$ And we must keep in mind the vasculardisrupting effect of electrochemotherapy, which could be further exploited with other specific treatment modalities.

\section{Conclusions}

Electroporation-based treatments are one success story of the translation of new technology into clinical practice. Electrochemotherapy with either bleomycin or cisplatin is effective in local tumour control of cutaneous and 
subcutaneous tumour nodules of different histologies, resulting in up to $\sim 75 \%$ long-term complete responses of treated tumours. After the progress of electrochemotherapy into broader clinical practice, next will be gene electrotransfer for gene therapy.

\section{Key educational points}

Electrochemotherapy is a local treatment which combines treatment with chemotherapeutic drugs and tissue electroporation, i.e. application of short, intense electric pulses to the tumour. Application of electric pulses potentiates the cytotoxicity of chemotherapeutic drugs by permeabilising the cell membrane of the cells at the site of electric pulse application, allowing for increased transmembrane transport of non-permeant or poorly permeant anticancer drugs, such as bleomycin or cisplatin, which possess high intrinsic cytotoxicity.

- The treatment of tumour nodules of different histologies, using appropriate instrumentation and procedures, results in $\sim 75 \%$ complete response of the treated nodules.

- The tumour should be adequately covered by the applied electric pulses; plate electrodes are designed for the treatment of superficial tumours, while needle electrodes are more suitable for exophytic or tumours lying deeper in the tissue.

- Electrochemotherapy can currently provide immediate clinical benefits in patients with multiple cutaneous and subcutaneous metastases which are not suitable for surgical removal.

- As well as in electrochemotherapy, electroporation is used in gene electrotransfer for gene therapy.

\section{Conflicts of interest}

The authors have no conflicts of interest.

\section{Acknowledgements}

The authors gratefully acknowledge financial support from the state budget of the Slovenian Research Agency and the European Commission's ESOPE project (QLK2002-02003), funded under the 5th Framework Programme. One of the authors (DM) would also like to thank Selma Corovic for her help in preparing Fig. 4.

\section{References}

1. Miklavcic D, Corovic S, Pucihar G, Pavselj N. Importance of tumour coverage by sufficiently high local electric field for effective electrochemotherapy. Eur J Cancer Suppl 2006;4:45-51.

2. Miklavcic D, Semrov D, Mekid H, Mir LM. A validated model of in vivo electric field distribution in tissues for electrochemotherapy and for DNA electrotransfer for gene therapy. Biochim Biophys Acta 2000;1523:73-83.
3. Pucihar G, Kotnik T, Valic B, Miklavcic D. Numerical determination of transmembrane voltage induced on irregularly shaped cells. Ann Biomed Eng 2006;34:642-52.

4. Mir LM. Bases and rationale of the electrochemotherapy. Eur $J$ Cancer Suppl 2006;4:38-44.

5. Mir LM, Belehradek M, Domenge C, et al. Electrochemotherapy, a new antitumor treatment: first clinical trial. CR Acad Sci III 1991;313:613-8.

6. Sersa G. The state-of-the-art of electrochemotherapy before the ESOPE study: advantages and clinical uses. Eur J Cancer Suppl 2006;4:52-9.

7. Byrne CM, Thompson JF. Role of electrochemotherapy in the treatment of metastatic melanoma and other metastatic and primary skin tumors. Expert Rev. Anticancer Ther 2006;6:671-8.

8. Pron G, Mahrour N, Orlowski S, et al. Internalisation of the bleomycin molecules responsible for bleomycin toxicity: a receptor-mediated endocytosis mechanism. Biochem Pharmacol 1999;57:45-56.

9. Orlowski S, Belehradek Jr J, Paoletti C, Mir LM. Transient electropermeabilization of cells in culture: increase in cytotoxicity of anticancer drugs. Biochem Pharmacol 1988;37:4727-33.

10. Sersa G, Cemazar M, Miklavcic D. Antitumor effectiveness of electrochemotherapy with cis-diamminedichloroplatinum(II) in mice. Cancer Res 1995;55:3450-5.

11. Sersa G, Cemazar M, Miklavcic D, Rudolf Z. Electrochemotherapy of tumours. Radiol Oncol 2006;40:163-74.

12. Mir LM, Devauchelle P, Quintin-Colonna F, et al. First clinical trial of cat soft-tissue sarcomas treatment by electrochemotherapy. $\mathrm{Br} J$ Cancer 1997;76:1617-22.

13. Tozon N, Sersa G, Cemazar M. Electrochemotherapy: potentiation of local antitumour effectiveness of cisplatin in dogs and cats. Anticancer Res 2001;21:2483-6.

14. Rols MP, Tamzali Y, Teissie J. Electrochemotherapy of horses: a preliminary clinical report. Bioelectrochem 2002;55:101-5.

15. Spugnini EP, Porrello A. Potentiation of chemotherapy in companion animals with spontaneous large neoplasms by application of biphasic electric pulses. J Exp Clin Cancer Res 2003;22:571-80.

16. Belehradek Jr J, Orlowski S, Ramirez LH, Pron G, Poddevin B, Mir LM. Electropermeabilization of cells and tissues assessed by the quantitative and qualitative electroloading of bleomycin. Biochim Biophys Acta 1994;1190:155-63.

17. Cemazar M, Milacic R, Miklavcic D, Dolzan V, Sersa G. Intratumoral cisplatin administration in electrochemotherapy: antitumor effectiveness, sequence dependence and platinum content. Anticancer Drugs 1998;9:525-30.

18. Sersa G, Cemazar M, Parkins CS, Chaplin DJ. Tumour blood flow changes induced by application of electric pulses. Eur J Cancer 1999;35:672-7.

19. Sersa G, Cemazar M, Miklavcic D, Chaplin DJ. Tumor blood flow modifying effect of electrochemotherapy with bleomycin. Anticancer Res 1999;19(5B):4017-22.

20. Gehl J, Skovsgaard T, Mir LM. Vascular reactions to in vivo electroporation: characterization and consequences for drug and gene delivery. Biochim Biophys Acta 2002;1569:51-8.

21. Sersa G, Krzic M, Sentjurc M, et al. Reduced blood flow and oxygenation in SA-1 tumours after electrochemotherapy with cisplatin. Br J Cancer 2002;87:1047-54.

22. Sersa G, Miklavcic D, Cemazar M, Belehradek Jr J, Jarm T, Mir LM. Electrochemotherapy with CDDP on LPB sarcoma: comparison of the anti-tumor effectiveness in immunocompetent and immunodeficient mice. Bioelectroch Bioener 1997;43:279-83.

23. Sersa G, Cemazar M, Menart V, Gaberc-Porekar V, Miklavcic D. Antitumor effectiveness of electrochemtherapy is inceased by TNF- $\alpha$ on SA-1 tumors in mice. Cancer Lett 1997;116:85-92.

24. Heller L, Pottinger C, Jaroszeski MJ, Gilbert R, Heller R. In vivo electroporation of plasmids encoding GM-CSF or interleukin-2 into existing B16 melanomas combined with electrochemotherapy induces long-term antitumour immunity. Melanoma Res 2000;10: 577-83. 
25. Mir LM, Glass LF, Sersa G, et al. Effective treatment of cutaneous and subcutaneous malignant tumours by electrochemotherapy. Br J Cancer 1998;77:2336-42.

26. Heller R, Jaroszeski M, Perrott R, Messina J, Gilbert R. Effective treatment of B16 melanoma by direct delivery of bleomycin using electrochemotherapy. Melanoma Res 1997;7:10-8.

27. Sersa G, Stabuc B, Cemazar M, Miklavcic D, Rudolf Z. Electrochemotherapy with cisplatin: clinical experience in malignant melanoma patients. Clin Cancer Res 2000;6:863-7.

28. Bloom DC, Goldfarb PM. The role of intratumour therapy with electroporation and bleomycin in the management of advanced squamous cell carcinoma of the head and neck. Eur J Surg Oncol 2005;31: 1029-35.

29. Byrne CM, Thompson JF, Johnston H, et al. Treatment of metastatic melanoma using electroporation therapy with bleomycin (electrochemotherapy). Melanoma Res 2005;15:45-51.

30. Marty M, Sersa G, Garbay JR, et al. Electrochemotherapy - an easy, highly effective and safe treatment of cutaneous and subcutaneous metastases: results of ESOPE (European Standard Operating Procedures of Electrochemotherapy) study. Eur J Cancer Suppl 2006;4: $3-13$.

31. WHO. WHO handbook for reporting results of cancer treatment. Vol 48. Geneva: WHO Offset Publications; 1997, p. 22-27.

32. Dev SB. Killing cancer cells with a combination of pulsed electric fields and chemotherapeutic agents. Cancer Watch 1994;3:12-4.

33. Mir LM, Gehl J, Sersa G, et al. Standard operating procedures of the electrochemotherapy: instructions for the use of bleomycin or cisplatin administered either systemically or locally and electric pulses delivered by the Cliniporator ${ }^{\mathrm{TM}}$ by means of invasive or non-invasive electrodes. Eur J Cancer Suppl 2006;4:14-25.

34. Domenge C, Orlowski S, Luboinski B, De Baere T, Belehradek Jr J, Mir LM. Antitumor electrochemotherapy: new advances in the clinical protocol. Cancer 1996;77:956-63.

35. Puc M, Corovic S, Flisar K, Petkovsek M, Nastran J, Miklavcic D. Techniques of signal generation required for electropermeabilization: survey of electropermeabilization devices. Bioelectrochemistry 2004; 64:113-24.

36. Pavselj N, Bregar Z, Cukjati D, Batiuskaite D, Mir LM, Miklavcic D. The course of tissue permeabilization studied on a mathematical model of a subcutaneous tumor in small animals. IEEE Trans Biomed Eng 2005;52:1373-81.
37. Snoj M, Cemazar M, Slekovec Kolar B, Sersa G. Effective treatment of multiple unresectable skin melanoma metastases by electrochemotherapy: case report. Croat Med J 2007;48:345-9.

38. Snoj M, Rudolf Z, Paulin-Kosir S, Cemazar M, Snoj R, Sersa G. Long lasting complete response in melanoma treated by electrochemotherapy. Eur J Cancer Suppl 2006;4(11):26-8.

39. Snoj M, Rudolf Z, Cemazar M, Jancar B, Sersa G. Successful sphincter-saving treatment of anorectal malignant melanoma with electrochemotherapy, local excision and adjuvant brachytherapy. AntiCancer Drugs 2005;16:345-8.

40. Shimizu T, Nikaido T, Gomyo H, et al. Electrochemotherapy of digital chondrosarcoma. J Orthop Sci 2003;8:248-51.

41. Glass LF, Fenske NA, Jaroszeski M, et al. Bleomycin-mediated electrochemotherapy of basal cell carcinoma. J Am Acad Dermatol 1996; 34:82-6.

42. Sersa G, Kranjc S, Cemazar M. Improvement of combined modality therapy with cisplatin and radiation using electroporation of tumors. Int J Radiat Oncol Biol Phys 2000;46:1037-41.

43. Kranjc S, Cemazar M, Grosel A, Sentjurc M, Sersa G. Radiosensitising effect of electrochemotherapy with bleomycin in LPB sarcoma cells and tumors in mice. BMC Cancer 2005;5:115.

44. Soden DM, Larkin JO, Collins CG, et al. Successful application of targeted electrochemotherapy using novel flexible electrodes and low dose bleomycin to solid tumours. Cancer Lett 2006;232:300-10.

45. Andre F, Mir LM. DNA electrotransfer: its principles and an updated review of its therapeutic applications. Gene Ther 2004;11(Suppl 1):33-42.

46. Cemazar M, Golzio M, Sersa G, Rols MP, Teissie J. Electrically-assisted nucleic acids delivery to tissues in vivo: where do we stand? Curr Pharm Des 2006;12:3817-25.

47. Heller LC, Heller R. In vivo electroporation for gene therapy. Hum Gene Ther 2006;17:890-7.

48. Edd JF, Horowitz L, Davalos RV, Mir LM, Rubinsky B. In vivo results of a new focal tissue ablation technique: irreversible electroporation. IEEE Trans Biomed Eng 2006;53:1409-15.

49. Nuccitelli R, Pliquet U, Chen X, et al. Nanosecond pulsed electric fields cause melanomas to self-destruct. Biochem Biophys Res Commun 2006;343:351-60.

50. Cemazar M, Parkins CS, Holder AL, Kranjc S, Chaplin DJ, Sersa G. Cytotoxicity of bioreductive drug tirapazamine is increased by application of electric pulses in SA-1 tumours in mice. Anticancer Res 2001;21:1151-6. 